\title{
Kompensation von Metallartefakten in der Computertomographie
}

\author{
D. Zerfowski \\ Institut für Algorithmen und Kognitive Systeme \\ Universität Karlsruhe \\ 76128 Karlsruhe \\ Email: zerfowsk@ira.uka.de
}

\begin{abstract}
Zusammenfassung. Metallische Fremdkörper wie z. B. Zahnfüllungen oder implantierte Schrauben wirken sich in computertomographischen Aufnahmen negativ auf die Bildqualität aus, indem sich streifenförmige Artefakte über große Bereiche des Bildes ausbreiten und für die Diagnostik relevante Informationen überdecken. Für eine zuverlässigere Befundung ist die Kompensation solcher Artefakte von besonderer Bedeutung. Das vorgestellte Verfahren lokalisiert in den gemessenen Rohdaten die durch das Metallobjekt verursachten Störungen, um diese mittels eines an die Geometrie des Tomographen angepaßten Verfahrens zu kompensieren.

Da das vorgestellte Verfahren unabhängig von nachfolgend angewandten Rekonstruktionsalgorithmen ist, handelt es sich um einen reinen Vorverarbeitungsalgorithmus, der sich einfach auf bereits existierenden Tomographieanordnungen implementieren läßt.
\end{abstract}

Schlüsselwörter: Computertomographie, Metallartefakte, Bildverbesserung, Radon-Transformation.

\section{$1 \quad$ Einleitung}

Die mathematisch-physikalische Grundlage der Computertomographie stellt die Radon-Transformation (Gleichung 1) dar [1, 2, 3]. Diese überführt die im durchstrahlten Objekt vorliegende Verteilung des Absorptionskoeffizienten $f(x, y)$ mittels Linienintegrale über alle möglichen, das Objekt durchquerenden Projektionsgeraden in eine Darstellung $g(l, \theta)$, wie sie in Abbildung 1 wiedergegeben wird. Dabei entspricht jeder Wert

$$
g(l, \theta)= \begin{cases}\int_{-\infty}^{\infty} f\left(\sqrt{l^{2}+z^{2}}, \theta+\arctan \left(\frac{z}{l}\right)\right) d z, & \text { falls } l \neq 0, \\ \int_{-\infty}^{\infty} f\left(z, \theta+\frac{\pi}{2}\right) d z, & \text { falls } l=0\end{cases}
$$

dem Wert des Linienintegrals entlang einer Projektionsgeraden mit dem Abstand $l$ vom Rekonstruktionszentrum und einem Normalenwinkel $\theta$ zur $x$-Achse.

Aufgrund des im Vergleich zum körpereigenen Gewebe hohen Absorptionskoeffizenten von Metallen werden eine erhebliche Anzahl von Messungen verfälscht. 
Abb. 1. Geometrische Interpretation der Radon-Transformierten.

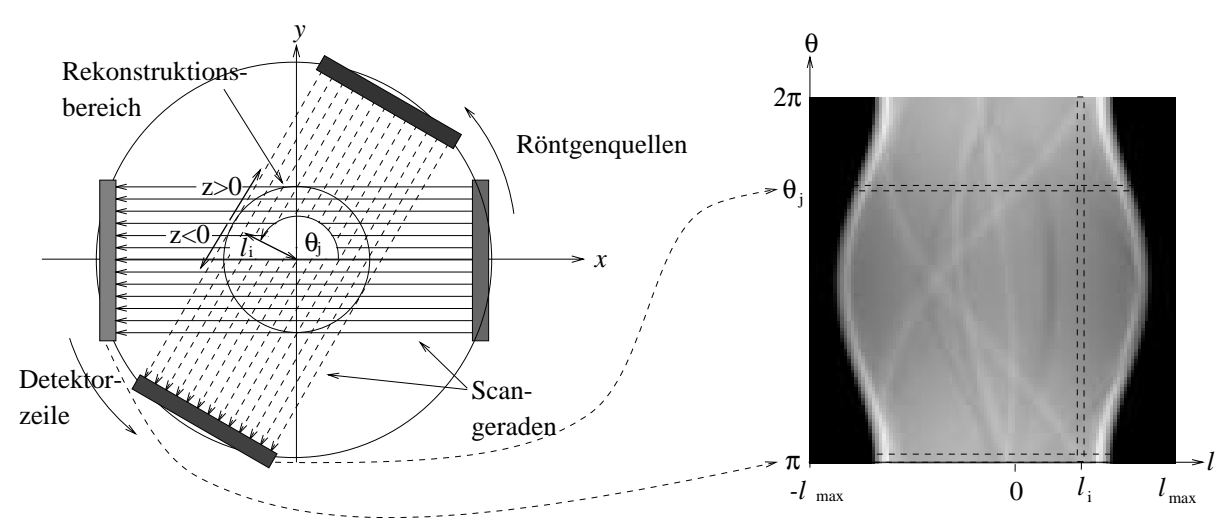

Ein in einer tomographischen Aufnahmeschicht liegendes, metallisches Objekt mit elliptischem Querschnitt (z.B. schräg durch die Aufnahmeschicht hindurchtretende Schraube) verursacht somit (Abb. 2 b) einen deutlich aus der restlichen Radon-Transformierten hervortretenden, sinusförmigen Streifen variabler Breite. Ohne vorherige Kompensation werden diese Fehler während des Rekonstruktionsprozesses über das gesamte Bild verteilt.

Kalender et al. [4] stellten ein halbautomatisches Verfahren vor, bei dem der Mediziner in den rekonstruierten Bilddaten das metallische Objekt manuell identifizieren muß. Mit diesen Angaben werden in der Radon-Transformierten die gestörten Daten gesucht, um nach einer nicht näher beschriebenen Korrektur und einer nochmaligen Rekonstruktion ein verbessertes Bild zu erhalten.

Wang et al. [5] wählen hingegen einen automatischen, dafür jedoch wesentlich rechenintensiveren Ansatz, indem sie ein an die Problemstellung angepaßtes, iteratives algebraisches Rekonstruktionsverfahren verwenden.

Das hier vorgestellte Verfahren sucht in der gemessenen Radon-Transformierten den Bereich der gestörten Daten unter Verwendung eines durch eine Histogrammanalyse vorgegebenen Schwellwertes. Aufgrund des endlichen Absorptionskoeffizienten von Metall, lassen sich die Ränder der gestörten Daten nicht exakt lokalisieren. Somit wird für alle Randpunkte über einen hinreichend großen Bereich in der Radon-Transformierten ein Schätzwert, basierend auf parallelen Projektionen, bestimmt. Hieraus ergibt sich für jeden Rotationswinkel $\theta$ (also einer Zeile in Abb. 1 rechts) ein guter Schätzwert für eine knapp am Metall vorbeiführende Messung.

Die anschließende Interpolation der in der Radon-Transformation verfälschten Daten nutzt die zuvor gemittelten Messungen, wobei neben parallelen auch nicht parallele Messungen entsprechend ihrer örtlichen Nähe im Objektbereich in die Schätzung eingehen.

Das Verfahren wurde in der Simulationsumgebung TomAS [6] implementiert und erprobt. 


\section{Kompensation in der Radon-Transformation}

Ausgehend von einer gemessenen Radon-Transformierten (Abb. 2 b) eines Phantoms mit einem Metallobjekt (helles kreisförmiges Objekt in Abb. 2 a), soll im folgenden das Kompensationsverfahren beschrieben werden.

In einem ersten Schritt wird eine Histogrammanalyse über die in der RadonTransformierten auftretenden Werte $g(l, \theta)$ durchgeführt. Hieraus ergibt sich eine untere Schranke für die durch die Metallkomponenten beeinflußten Projektionen. Unter Verwendung des so bestimmten Schwellwertes wird zeilenweise in der Radon-Transformierten der linke $\left(d_{\mathrm{l}}(\theta)\right)$ und rechte $\left(d_{\mathrm{r}}(\theta)\right)$ Rand des Metall jeinflusses lokalisiert.

Für eine zeilenweise lineare Interpolation der Werte $g(d, \theta)$ für $d_{1}<d<$ $d_{\mathrm{r}}$ werden nicht direkt die Werte $g\left(d_{\mathrm{l}}, \theta\right)$ und $g\left(d_{\mathrm{r}}, \theta\right)$ verwendet. Um lokale Schwankungen in der Radon-Transformierten auszugleichen, wird als Schätzung für eine knapp außerhalb des Metalls verlaufende Projektion eine Mittelung über Projektionen durchgeführt, die in einem $3 \mathrm{~mm}$ breiten Streifen parallel verlaufen. Formal ergibt sich für die Interpolation der gestörten Daten $g(d, \theta)$ mit $d_{1}(\theta)<$ $d<d_{\mathrm{r}}(\theta)$ und $P$ der Anzahl der zu mittelnden Geraden

$$
\begin{gathered}
\bar{g}(d, \theta)=\frac{d-d_{\mathrm{l}}(\theta)}{d_{\mathrm{r}}(\theta)-d_{\mathrm{l}}(\theta)} m_{\mathrm{l}}(\theta)+\frac{d_{\mathrm{r}}(\theta)-d}{d_{\mathrm{r}}(\theta)-d_{\mathrm{l}}(\theta)} m_{\mathrm{r}}(\theta) \text { mit } \\
m_{\mathrm{l}}(\theta)=\frac{1}{P} \sum_{i=0}^{P-1} g\left(d_{\mathrm{l}}-i \cdot \Delta d, \theta\right) \text { und } m_{\mathrm{r}}(\theta)=\frac{1}{P} \sum_{i=0}^{P-1} g\left(d_{\mathrm{r}}+i \cdot \Delta d, \theta\right) .
\end{gathered}
$$

Bereits diese einfache Interpolationsmethode liefert nach der Rekonstruktion eine deutlich verbesserte Bildqualität. Jedoch weißt die Radon-Transformierte im Bereich der interpolierten Daten deutliche horizontale Strukturen auf. Sie verläuft somit in $\theta$-Richtung nicht glatt. In Abbildung $2 \mathrm{~d}$ ) ist ein entsprechender Ausschnitt aus der Radon-Transformierten wiedergegeben. Aus dem genannten Grund wird hier eine weitere Interpolationsmethode vorgestellt.

Die Erweiterung der zuvor beschriebenen eindimensionalen Interpolation in die zweite Dimension, also in $\theta$-Richtung, durch Verwendung einer einfachen zweidimensionalen Filterung auf der Radon-Transformierten ist nicht von Vorteil. Der Grund hierfür liegt darin, daß für festes $d$ bei kleinen Veränderungen in der $\theta$-Koordinate, bereits über Daten gemittelt würde, die sich entlang von Projektionsgeraden ergeben, die das Metallobjekt in einem relativ großen Abstand passieren. Es würde somit über Daten gemittelt, die wenig Informationen aus der örtlichen Nähe des metallischen Fremdkörpers enthalten. Dieses gilt in besonders starkem Maße für Metallobjekte, die vom Rekonstruktionszentrum weit entfernt liegen.

Wünschenswert ist es also für jede durch $(d, \theta)$ festgelegte Gerade, die den im Metall liegenden Punkt $(x, y)$ durchläuft, alle die Projektionsgeraden in der Schätzung zu berücksichtigen, die mit einem geringen Abstand zu $(x, y)$ außerhalb des Metalls verlaufen und außerdem nahezu parallel zu $(d, \theta)$ liegen.

Die Einschränkung auf nahezu parallele Geraden und damit auf kleine Variationen von $\theta$ bewirkt, daß lediglich Projektionsgeraden berücksichtigt werden, 
Abb. 2. Metallartefakte in der Radon-Transformierten und Rekonstruktion.

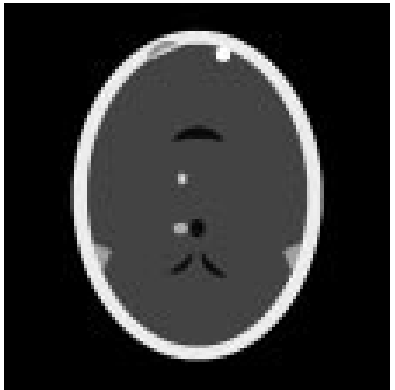

a) Phantom mit Metall

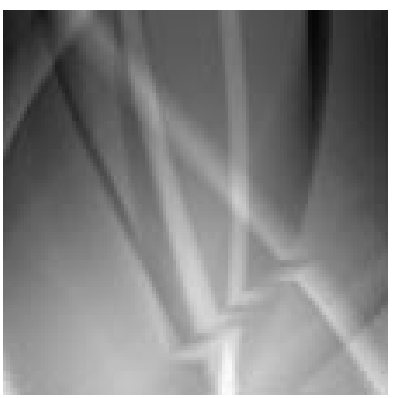

d) Horizontale Strukturen bei zeilenweiser Interpolation

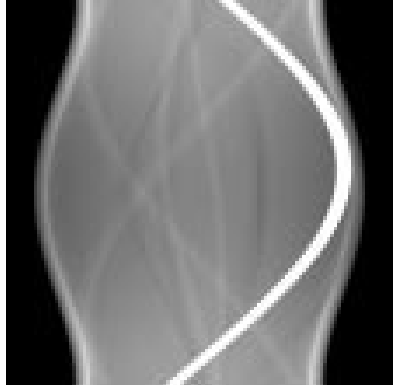

b) Radon-Transformierte von a)

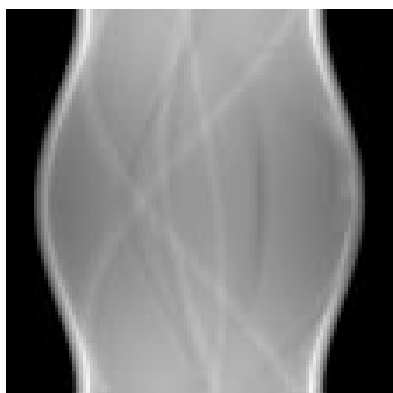

e) Geometrieangepaßte Interpolation

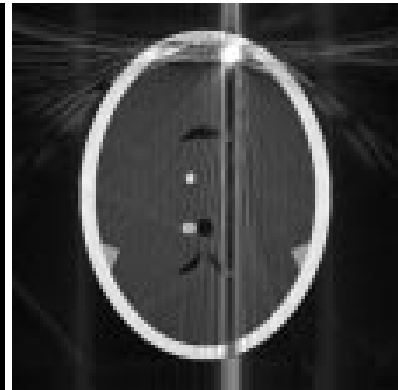

c) Rekonstruktion ohne Kompensation

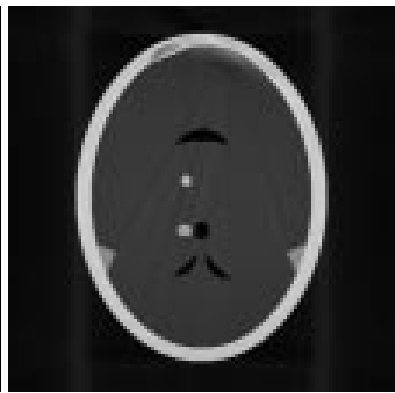

f) Rekonstruktion nach Kompensation

die auf ihrem Weg durch das Objekt eng benachbarte Gebiete durchlaufen und damit eine hohe Korrelation zueinander besitzen. Geht man von einem inkrementellen Rotationswinkel $\Delta \theta$ zwischen zwei, sich im Rekonstruktionszentrum schneidenden Geraden aus, so besitzen die zwei Punkte, die mit einem radialen Abstand $r$ vom Ursprung auf den Geraden liegen, einen Abstand

$$
a=2 r\left|\sin \left(\frac{\Delta \theta}{2}\right)\right| .
$$

Für den in den Simulationen verwendeten Paralleltomographen mit 256 Sichten und Detektoren bedeutet dies, daß die Positionen des auf der durch den Ursprung verlaufenden Projektionsgeraden und dem Rand des Rekonstruktionsbereiches (mit dem Durchmesser $2 r=300 \mathrm{~mm}$ ) liegenden Punktes zwischen zwei Sichten einen Abstand von $\approx 1,84 \mathrm{~mm}$ besitzen. Nach vier Sichten beträgt der entsprechende Abstand bereits $\approx 7,4 \mathrm{~mm}$. Mit einem größer werdenden Rotationswinkel durchlaufen diese Gebiete fast vollständig verschiedene Regionen innerhalb des Objektes (mit Ausnahme ihres Schnittpunktes), und eine entsprechende Mittelung ergibt keine Vorteile.

Die geometrieangepaßte Bestimmung der für die Korrektur der gestörten Daten $g(d, \theta)$ benötigten Werte in der Radon-Transformierten ergibt sich unter 
Berücksichtigung des Gesagten wie folgt (Abb. 2 e).

- Analog zur zeilenweisen Interpolation, bestimme über einen mittels einer Histogrammanalyse bestimmten Schwellwert den linken und rechten Rand $\left(d_{1}(\theta)\right.$ und $\left.d_{\mathrm{r}}(\theta)\right)$ der gestörten Daten in der Radon-Transformierten.

- Analog zur zeilenweisen Interpolation (Gleichung 3), berechne die Mittelwerte $\mathrm{m}_{\mathrm{l}}(\theta)$ und $\mathrm{m}_{\mathrm{r}}(\theta)$ außerhalb der beiden Ränder, linear gewichtet mit dem Kehrwert ihres Abstands vom Rand.

- Schätze den Wert $\bar{g}(d, \theta)$ mit $d_{1}(\theta)<d<d_{\mathrm{r}}(\theta)$ gemäß

$$
\begin{aligned}
\bar{g}(d, \theta)= & \sum_{\theta^{\prime}=\theta-j \cdot \Delta \theta}^{\theta+j \cdot \Delta \theta} \frac{1}{1+\left|\theta^{\prime}-\theta\right|} \cdot \\
& \left(\frac{d-d_{\mathrm{l}}\left(\theta^{\prime}\right)}{d_{\mathrm{r}}\left(\theta^{\prime}\right)-d_{\mathrm{l}}\left(\theta^{\prime}\right)} \cdot \mathrm{m}_{\mathrm{l}}\left(\theta^{\prime}\right)+\frac{d_{\mathrm{r}}\left(\theta^{\prime}\right)-d}{d_{\mathrm{r}}\left(\theta^{\prime}\right)-d_{\mathrm{l}}\left(\theta^{\prime}\right)} \cdot \mathrm{m}_{\mathrm{r}}\left(\theta^{\prime}\right)\right),
\end{aligned}
$$

wobei die Anzahl $j$ der bei der Interpolation berückichtigten unterschiedlichen Winkel einmalig der verwendeten Scannergeometrie angepaßt werden muß.

Im Idealfall ist nach der zuvor beschriebenen Kompensation im rekonstruierten Bild das metallische Objekt nicht mehr erkennbar (Abb. 2 f). Um den Bereich des kompensierten Metalls im Bild zu markieren, können die Randkurven $d_{\mathrm{l}}(\theta)$ und $d_{\mathrm{r}}(\theta)$ ausgenutzt werden. Aufgrund ihres Verlaufes in der RadonTransformierten läßt sich die Örtlichkeit des Metallobjektes bestimmen und als zusätzliches Überlagerungsbild in das rekonstruierte Bild einblenden. Für eine detaillierte Beschreibung der Korrespondenzen zwischen den Randkurven und Strukturen im rekonstruierten Bild sei auf [7] verwiesen.

Danksagung. Wir danken der Deutschen Forschungsgemeinschaft für die Unterstützung durch den Sonderforschungsbereich SFB 414 „Informationstechnik in der Medizin - Rechner und sensorgestützte Chirurgie“ (Teilprojekt Q1).

\section{Literatur}

1. G. T. Herman: Image reconstruction from projections. Academic Press, 1980.

2. F. Natterer: The Mathematics of Computerized Tomography. Wiley, 1986.

3. A. C. Kak und M. Slaney: Principles of Computerized Tomography. New York: IEEE Press, 1987.

4. W. A. Kalender, R. Hebele und J. Ebersberger: Reduction of CT artifacts caused by metallic implants. Radiology, 164(2):576-577, 1987.

5. G. Wang, D. L. Snyder, J. A. O'Sullivan und M. W. Vannier: Iterative Deblurring for CT Metal Artifact Reduction. IEEE Transactions on Medical Imaging, 15(5):657, Oktober 1996.

6. D. Zerfowski, T. Rohlfing, U. Mende und Th. Beth: TomAS - Tomographic Algorithms and Ultrasound Simulation. In: Lemke (Herausgeber): CAR'97, Seite 1017. Elsevier Science, 1997.

7. D. Zerfowski: A new Method for Motion Artifact Compensation in CT. In: Proceedings Medical Imaging, San Diego, California, USA, 1998. SPIE. 